\title{
Editorial $\quad$ The Future Hospital: A Business Architecture View
}

\section{Ariffin Marzuki MoKHTAR}

Submitted: 5 Apr 2017

Accepted: 8 May 2017

Online: 26 Oct 2017
Directors Office, Hospital Universiti Sains Malaysia, Jalan Sultanah

Zainab II, 16150 Kubang Kerian, Kelantan, Malaysia

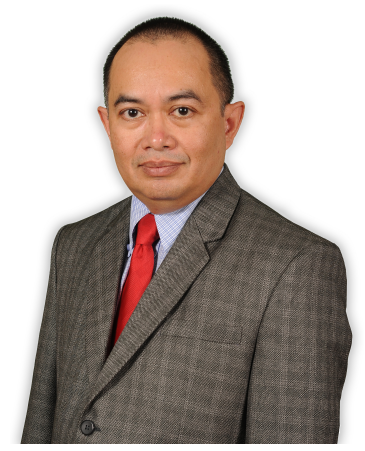

To cite this article: Mokhtar AM. The future hospital: A business architecture view. Malays J Med Sci. 2017;24(5): 1-6. https://doi.org/10.21315/mjms2017.24.5.1

To link to this article: https://doi.org/10.21315/mjms2017.24.5.1

\begin{abstract}
The future hospital is a resilient, physical learning facility featuring digital enhancement and leveraging an ecosystem of platforms for the Internet of Things (IoT) and analytics, achieving patient-centric care delivery via multidisciplinary healthcare provider teams coordinated to meet patients' medical, psychological, social and economic needs. It exists in a just ecosystem that assimilates the care spectrum from healthy living, the prevention of disease to acute care and the rehabilitation of patients recuperating from illnesses. It will take some time for these future hospitals to be built or for current hospitals to evolve and/or transform, but efforts to spread wisdom among the stakeholders, healthcare providers and patients must start now. The development of the digital components can also begin today, as can competency building for the healthcare providers who will be staffing these future hospitals, ensuring that they are equipped with competent staff employing patient-centric care processes that cater to patients' current and future needs.
\end{abstract}

Keywords: future hospital, technology, information system, information communication technology, business architecture, patient safety 
'The future is already here-it's just not evenly distributed' (1).

\section{Introduction}

A number of articles have been written about the future hospitals worldwide. The discussions and findings includes visions, roles, competency, capabilities, guidelines, and an architect's perspective $(2,3,4,5,6,7,8)$. The Royal College of Physician, in the United Kingdom triennially publishes the Future Hospital Journal to provide a forum for debate around the delivery of secondary healthcare (9). What are the characteristics of these future hospitals, and what are the challenges facing an existing hospital that wants to morph into a future hospital in Malaysia? The expectation for the future hospital is that it will have the capability to solve current problems, will cater to patients' ever-changing future needs and it will enable healthcare providers to deliver high quality and safe care to their patients.

\section{Background}

Modern hospitals are very different from the cramped vermin-infested institutions of the past. Laws and regulations have made significant strides in ensuring the safety and comfort of patients admitted to the modern hospital. Technology filled hospitals are available in many parts of the world including Malaysia, and they have served many patients over the years. Issues still present themselves in the form of rising healthcare delivery costs, questionable sustainability of the organisations delivering healthcare, out-dated business models and, patient safety concerns, such as hospital-acquired infections, medical errors and healthcare providers' ethics. The Committee on the Quality of Healthcare highlighted each of these in its first report (10). There are also unmet needs among the patients, care-takers, families and the community such as autonomy in therapy, informed consent and adverse incident disclosure (11).

In 2001, the Committee's second report, 'Crossing the Quality Chasm: A New Health System for the 21st Century' was pivotal in providing a strategic direction for the design of a 21st century healthcare delivery system. The report addresses quality-related issues more broadly, providing a strategic direction for redesigning the healthcare delivery system of the 21st century. Fundamental reform of healthcare is needed to ensure that all (patients) receive care that is safe, effective, patient centred, timely, efficient and equitable (12). The report also set the four stages of evolution for the design of health organisation based on three sets of dimensions: the patient's experience, knowledge and skills management and care delivery. The most notable points in these stages is the patientcentricity of the design and the information-rich clinical environment that stresses competency building, a multidisciplinary approach to patient care and coordinated care with information technology as the basic building blocks for making systems work, tracking performance and increasing learning (12). The report sets the foundation for future hospitals to build upon the premise that they will have the capability to solve the current problems, cater to the everchanging future needs of the patients and enable healthcare providers to deliver high quality and safe care.

This editorial's aims to discuss, from a business architecture point of view, what a future hospital is, why it is a needed, where and when shall it operate, capabilities, competencies, roles and responsibilities involved in the future hospital, and how to begin the journey toward creating a future hospital that will cater to the current and future patients' needs including a staff empowered to deliver high quality and safe care.

\section{The Future Hospital}

From the start, the future hospital will be designed to be patient centric, with healthcare providers working in high performing multidisciplinary teams who would look into the patients' medical, nursing, psychological and socio-economic needs. Its goal, by design, is to provide a safe and healing environment for patients, delivering high quality services across the healthcare spectrum. The hospital's design will consider the strategy, people, structure, rewards and processes related to it (13). The strategies for adoption by the future system include clinician-hospital alignment, quality, patient safety, efficiency through productivity and financial management, integrated information systems, integrated providers networks, engaged employees and physicians, strengthened finances, payer-provider partnership, scenario-based planning and population health improvement (5). The people 
operating the future hospital must be competent to cater to the current and future needs of both the patients and staff alike. A competent staff has knowledge and mastery of its subject matter and engages in continuous improvement methods, building the skills needed to use the tools of their trades (including digital tools) effectively and the ability to self-motivate for lifelong learning (14). The competencies of future hospitals include, but not limited to, the design and implementation of patient-centered integrated care, the creation of accountable governance and leadership, strategic planning in an unstable environment, internal and external collaboration, financial stewardship and enterprise risk management, the engagement of full employee potential and the collection and utilisation of electronic data for performance improvement are also part of the system (5).

Future hospitals will operate based on a mindful infrastructure for high reliability that includes the capability to discover unexpected events (mindful anticipation) and manage them (mindful containment). This mindfulness will lead to high reliability with five characteristics that define these institutes: pre-occupation with failure, reluctance to simplify, sensitivity to operations, commitment to resilience and deference to expertise (15). The hospitals reward system will be value-based, which is defined as outcomes attained over the costs incurred. The multi-disciplinary team rewards will be based on outcomes and performance for excellence across the board, with transparent measures based on continuous feedback on both tangible and intangible indicators (16). Processes will be managed across the enterprise using the best of practices that are continuously measured through feedback on their design and execution dimensions, and compliance is assured via a closed-loop mechanism (17). Information and communication technology will play an important role in the future hospital as a tool for delivering high quality, safe and patient-centric care by allowing partnerships between patients and providers including the co-ordination of multi-disciplinary teams of care providers across the care spectrum in a decentralised manner (18).

\section{Patients' Digital Engagement}

The future hospital's recipe for success will rely on engaging of patients as a part of the multidisciplinary healthcare team in one secure borderless ecosystem that fosters the facilitation and enablement of clinical services across the hospital, community and patients' home settings. This is a huge paradigm shift for both patients and healthcare providers, that will necessitate a competency building programme to allow both groups to gain the knowledge, skills and attitudes they need to grow their partnership and for it to flourish in time.

The hospital's management team must take a facilitative and enabling role (using a leadership platform) that promotes this shift, to ensure its sustainability by conducting programmes for both healthcare providers and patients. Information and communication technology will play a vital role in this partnership, and an ecosystem of platforms must be designed to allow the partnership to be executed in a digital platform designed for patient partnership (19). Here, the patient will have access to and will input data on his or her health and symptoms and the information will be available to each of his or her healthcare providers. This digital platform can also be escalated into a lifeline, if the patient is in dire need of urgent care. This lifeline will not only be digitally available, but also physically present in the chain of survival that extends beyond the hospital to the community and homes via a network of healthcare outreach providers. Patients' homes will serve as a hub for many sensors related to their wellbeing (leveraging the technology of the IoT in patient care) and will function, when needed, as an extension of the hospital with provisions for the escalation of care by using outreach teams who shall be made available to assist them should the need arise (18). These healthcare providers will work seamlessly as part of a multidisciplinary team that uses another platform, one for clinical care delivery, allowing for collaboration among team members from many disciplines.

The talent management platform will serve as another facilitation to leadership, patient and care platforms that allow collaborations to occur in a knowledge-sharing ecosystem. Each of these platforms will be supported by a comprehensive, secure and borderless technology infrastructure that allows it to work seamlessly across platforms and care settings. It will also serve as a coordination point for the physical resources needed for such partnership to occur. This is what it means when we say that the future hospital will have both a physical and digital presence; each will compliment the other, allowing the patient meaningful access to his or 
her health and treatment information across the care spectrum. Patient partnerships, knowledge sharing and the co-ordination of resources based on the patient's needs in a resilient environment, will ensure that care is delivered not only safely but also to a high standard. This quality will be measurable by the indicators from each activity and data from sources close to the patient care transactions, thus allowing analytics to be used continuously to monitor the data and transform it into decisions and actionable care for patients across the whole spectrum of the healthcare ecosystem.

\section{Challenges for Establishing the Future Hospital in Malaysia}

There are two challenges in designing a new or evolving an existing Malaysian hospital to be a hospital of the future. The first is having the capability to design a new future hospital from scratch, and the second is the capability to evolve current hospitals to meet these new standards (20). These capabilities can be broken down into capability increments to address the challenges. The increments can be further be divided into three dimensions that include processes, people and materials.

The process dimension is first catered for by defining the concepts, business processes and information management needed for the future hospital. The people dimension includes individual and collective training efforts, plus professional development for healthcare providers and non-clinical or managerial staff. The material dimension includes the infrastructure information, technology and equipment in the hospital that the healthcare providers will use. Using the above framework, a roadmap for a future hospital can be devised for both capabilities. In order to design a future hospital, a team must first have the competency to plan, build, run and monitor the overall initiative (21). This competency can be built individually, or via a collective programme that leads to the team's professional development. This programme will enable the team to design the concept of the future hospital and its business processes, including its information management plans. Only then can the materials for the projects be defined including the infrastructure, information technology and equipment identified. These will be used to estimate the cost to build both the physical building and digital entities (20).
Evolving an existing hospital into a future hospital will require a different methodology as the existing hospitals will be deemed a legacy system that shall require to a new systems (22). An assessment of the hospital's core, operating environment and support environment must be done to determine the extent of change that is required and then factored into the evolution initiative.

The second step with a legacy system is similar to the design of a new hospital elaborated above: the future systems are the architecture targets for the core, target architecture for the core, operating and support environments, all of which will need to be designed and built based on the insights gained from the assessment of the legacy system incorporating its systems engineering, management and project activities (along with its key processes and products) and taking into consideration the technology that is available both now and in the future.

All of these plans must be executed as an evolution or transformation programme over time, with milestones and deliverables to guide them. Both can be used as examples for any organisation in Malaysia seeking either to design or evolve into a hospital of the future.

\section{Conclusion}

The future hospital is not about the physical structure/technology; it is about building staff competency, creating a just ecosystem that consists of platforms for patient engagement, staff talent management and associated business services supported by technology by design. The future hospital is about delivering high quality, safe, efficient and effective care. It is also about the engagement and facilitation of patients' interactions with their healthcare providers. Finally, it is about employing innovative new methods to re-invent the care processes to serve the patient population.

In summary, the future hospital is a digital facility that humanises healthcare for patients in the quest of cures for ailments. It is a highly reliable organisation that is resilient, manages knowledge and is just to the healthcare providers, including the stakeholders (23, 24). It exists as an important component of an ecosystem that promotes the full spectrum of healthcare services in the community it serves, and it is centred on the digital home and family units it supports. These are the winning characteristics of the future hospital that will 
enable it to cater to current and future patients' needs.

\section{Acknowledgements}

I wish to thank Dr Nor Zainiyah Norita binti Mokhtar from the Kuliyyah of Languages and Management, International Islamic University of Malaysia, for editing this manuscript.

\section{Author's Contributions}

Conception and design, drafting of the article and final approval of the article.

\section{Correspondence}

Dr Ariffin Marzuki Mokhtar

BMedSc, MD, MMed (Anaest), UKM

CITA-A (Business Architecture), TOGAF 9.1 and

COBIT 5 Certified

Deputy Director (Operations) Hospital Universiti

Sains Malaysia and Senior Lecturer

Department of Anaesthesiology, School of Medical

Sciences, Universiti Sains Malaysia,

16150 Kubang Kerian, Kelantan, Malaysia.

Tel: 609-7673005/3002

E-mail: ariffinm@usm.my

\section{References}

1. Gibson W. Not so big in Japan. The Economist. December 4, 2003. Available from: www. economist.com/node/3252464

2. Future Hospital Commission. Future hospital: caring for medical patients. A report from the Future Hospital Commission to the Royal College of Physicians. London: Royal College of Physicians; 2013. eISBN 9781860165191

3. TIME. Inside the hospital room of the future [Video file]; 2015. Available from: https://www. youtube.com/watch?v=JIICZCOZJkk

4. Ribera J, Antoja G, Rosenmoller M, Pablo Borras P. Hospital of the future: a new role for leading hospitals in Europe. Barcelona: IESE Business School; 2016. 74-90p. Report no ST-388-E

5. American Hospital Association. 2011 Committee on Performance Improvement, Jeanette Clough, Chairperson. Hospitals and care systems of the future. Chicago: American Hospital Association; 2011. 7p. Available from: http://www.aha.org/ about/org/hospitals-care-system-future.shtml
6. Gamble, M. The future of healthcare: 9 capabilities for post reform success. USA; 2012. Available from: http://www. beckershospitalreview.com/healthcareinformation-technology/the-new-age-ofhealthcare-9-capabilities-for-post-reform-success. html

7. The Joint Commission. Healthcare at the crossroads: guiding principles for the development of the hospital of the future (Internet). USA: The Joint Commission; 2008. Available from: https://www.jointcommission. org/assets/1/18/Hospital_Future.pdf

8. de Grey S. Designing the future hospital: an architect's perspective. Future Hospital Journal. 2015;2(1):15-18. https://doi.org/10.7861/ futurehosp.15.007

9. Future Hospital Journal. UK: Royal College of Physician. Available at: http://futurehospital. rcpjournal.org

10. Kohn LT, Corrigan JM, Donaldson MS, editors. To err is human: building a safer health system. Committee on Quality of Healthcare in America, USA: Institute of Medicine, National Academies Press; 200o. Available from: http://www.nap. $\mathrm{edu} /$ catalog/9728.html

11. Holland DE, Mistiaen P, Bowles KH. Problems and unmet needs of patients discharged "home to self-care". Professional Care Management. 2011;16(5):240-250. https://doi.org/10.1097/ NCM.obo13e31822361d8

12. Committee on Quality Healthcare in America. Crossing the quality chasm: a new healthcare system for the 21st century. Institute of Medicine, USA: National Academies Press; 2001. Available from: http://www.nap.edu/catalog/10027.html

13. Galbraith JR. Designing organizations. 3rd ed. San Francisco (CA): Wiley; 2014. ISBN 978-1-11846382-6 (epub).

14. Pralahad CK, Hamel G. The core competence of the corporation. Harvard Business Review MayJune 1990. Available from: www.hbr.org

15. Weick KE, Sutcliffe KM. Managing the unexpected. Resilient performance in an age of uncertainty. 2nd ed. San Francisco (CA): Wiley; 2007. 
16. Porter ME, Teisberg EO. Redefining health care. Creating value based competition on results. Boston (MA): Harvard Business School Press; 2006.

17. Kaplan RS, Norton DP. Mastering the management system. Harvard Business Review Jan 2008. Available from: www://hbr.org/ Reprint Ro801D

18. Rajakulendran N, MacIntosh E, Salah H, Khayat K. Transforming health: toward decentralized and connected care [Internet]. Canada: MaRS; 2014. Available from: https://www.marsdd.com/newsand-insights/transforming-health-decentralizedconnected-care/

19. LeHung H, Howard C, Gaughan D, Logan D. Building a digital business technology platform. US: Gartner; 2016. Report ID Go0297286.

20. The Open Group. The Open Group Architecture Framework @ version 9.1. (C) Open Group Standard G116. US: Open Group; 2011. Available from: www.opengroup.org/
21. Controlled Objective for Business and Information Technology version 5 (COBIT $\AA$ ) 5). US: ISACA; 2012. ISBN 978-1-60420-237-3. Available from: www.isaca.org/

22. Bergey JK, Northrop LM, Smith DB. Enterprise framework for the disciplined evolution of legacy systems. Pittsburg (PA): Carnegie Mellon University; 1997. Technical Report CMU/SEI-97TR-007, ESC-TR-97-007.

23. Leape LL, Shore MF, Dienstag JL, Mayer RJ, Edgman-Levitan S, Meyer GS, et al. Perspective: a culture of respect, Part 1: The nature and causes of disrespectful behavior by physicians. Acad Med. 2012;87:845-852. https://doi.org/10.1097/ ACM.obo13e318258338d

24. Leape LL, Shore MF, Dienstag JL, Mayer RJ, Edgman-Levitan S, Meyer GS, et al. Perspective: a culture of respect, Part 2: Creating a culture of respect. Acad Med. 2012;87:853-858. https:// doi.org/10.1097/ACM.obo13e3182583536 